\title{
Conflits d'usage et de voisinage autour de la ressource en eau. Illustration à partir du littoral charentais
}

Land-use conflicts about water resources. The case of the coastal area of PoitouCharentes

Luc Bossuet et Ornella Boutry

\section{OpenEdition}

\section{Journals}

Édition électronique

URL : http://journals.openedition.org/economierurale/3680

DOI : 10.4000/economierurale.3680

ISSN : 2105-2581

\section{Éditeur}

Société Française d'Économie Rurale (SFER)

Édition imprimée

Date de publication : 30 novembre 2012

Pagination : 74-87

ISSN : 0013-0559

Référence électronique

Luc Bossuet et Ornella Boutry, "Conflits d'usage et de voisinage autour de la ressource en eau. Illustration à partir du littoral charentais », Économie rurale [En ligne], 332 | novembre-décembre 2012, mis en ligne le 30 décembre 2014, consulté le 30 avril 2019. URL : http://journals.openedition.org/ economierurale/3680 ; DOI : 10.4000/economierurale.3680 


\section{Conflits d'usage et de voisinage autour de la ressource en eau Illustration à partir du littoral charentais}

LUC BOSSUET 1 • Agroparistech, UMR INRA SADAPT, Paris

Ornella BOUTRY2 • CRIEF-TEIR, EA 2249, Université de Poitiers

D nombreux territoires sont aujourd'hui marqués par des problèmes de coordination entre des collectifs d'acteurs autour de l'usage d'une ressource spatialisée, qui peuvent déboucher sur des conflits d'usage et de voisinage. Ce type de conflit met ainsi en scène des acteurs ou des activités en situation de concurrence ou reliés par des phénomènes d'externalités. L'eau, en tant que bien collectif, est une ressource de plus en plus convoitée pour de multiples usages. C'est particulièrement le cas le long des littoraux et des espaces estuariens du fait des interdépendances entres les acteurs et de leur proximité spatiale (Torre et al., 2006 ; Bossuet et Torre, 2009).

Nous avons choisi de traiter la question de la gestion quantitative de la ressource en eau sur le littoral picto-charentais. Les caractéristiques de ce cas d'étude sont en effet particulièrement pertinentes au regard de notre problématique : du fait des nombreuses et graves crises quantitatives qui marquent le territoire depuis les années 2000, en lien avec le décalage important observé entre la disponibilité de la ressource en eau et les besoins anthropiques, le littoral picto-charentais apparaît comme l'un des territoires français les plus conflictuels en ce qui concerne l'usage quantitatif de la ressource. La ressource en eau est par ailleurs mobilisée par plusieurs activités économiques jouant un rôle central dans le dynamisme économique du territoire (Bouba-Olga et al., 2009 ; Boutry, 2011). Nous avons identifié plusieurs dysfonctionnements, impliquant majoritairement trois collectifs : deux secteurs productifs primaires locaux que sont l'agriculture et la conchyliculture, et l'activité de plaisance. Les deux premiers sont en situation de concurrence sur l'utilisation de la ressource en eau. Plus récemment, les conchyliculteurs s'opposent également aux plaisanciers, cette fois au niveau de l'occupation de l'espace maritime. La méthodologie développée pour comprendre la situation conflictuelle sur le territoire repose sur trois axes d'investigation : la Presse quotidienne régionale $(\mathrm{PQR})^{1}$, les enquêtes à dires d'experts ${ }^{2}$ et le recueil de documents et d'informations d'ordre technique capables de renseigner le discours des acteurs rencontrés. Ces trois éléments complémentaires permettent d'établir une cartographie de la conflictualité du territoire d'étude.

La situation de conflit est appréhendée comme un moment particulier dans un processus continuel de coordination (Torre et Caron, 2005), dont le but est de définir un nouvel équilibre entre les forces en présence. De plus, les conflits d'usage et de voisinage possèdent une forte inscription

1. Une base de données des conflits a été établie à partir d'articles de journaux de l'édition CharenteMaritime du quotidien «Sud-Ouest » de 2005 (301 articles recensés et 228 conflits). Deux faits importants ont été mis en évidence : le conflit autour de la ressource en eau est le plus médiatisé et les conflits sont majoritairement des conflits entre collectifs d'acteurs.

2. Une cinquantaine d'enquêtes ont été réalisées auprès d'acteurs du territoire (institutionnels, professionnels, associations, gestionnaires, ...) pour obtenir leur perception de la situation et leurs connaissances. 
spatiale, d'où la nécessité d'introduire la dimension territoriale à l'étude des défauts de coordination qu'ils sous-tendent. Nous proposons, dans cette perspective, d'analyser les conflits d'usage et de voisinage, en nous interrogeant sur l'origine des rivalités, sur leurs modes d'expression et sur les possibilités de résolution, dans un but de développement territorial. L'approche que nous développons dans cette étude est issue d'une réflexion croisée sur le lien entre proximité et conflit, empruntant à la fois à la sociologie et à l'économie.

Il est ainsi possible d'identifier une tradition sociologique qui considère la conflictualité comme un élément constitutif de la socialisation, plutôt que comme une simple perturbation du système social (Simmel, 1981 [1908]). Plus particulièrement, les analyses sociologiques portant sur les mécanismes de conflictualité en lien avec la proximité spatiale se sont initialement développées à partir de recherches sur les antagonismes de classes en milieu urbain. Elles soulignent le fait que la conflictualité dans un contexte de proximité spatiale s'explique en partie par le degré de cohésion interne des groupes sociaux, cohésion qui peut à son tour être reconfigurée par la dynamique propre des conflits (Elias et Scotson, 1994 [1965]) ${ }^{3}$. Par ailleurs, la proximité spatiale peut être un facteur direct par lequel se différencie l'identité d'un groupe par rapport à un autre où se reconfigure sa cohésion interne, alors que la plupart des caractéristiques sociales qui le définissent tendraient à gommer sa spécificité, s'il n'était pas inscrit dans cette confrontation que lui impose cette proxi-

3. "Un groupe est doté d'un degré de cohésion (cohesion rate) plus élevé que l'autre et ce différentiel d'intégration contribue substantiellement au surplus de pouvoir de ce premier groupe; sa cohésion plus forte lui permet de réserver au profit de ses membres des positions sociales avec un potentiel de pouvoir plus élevé et d'une nature différente, ce qui renforce à son tour sa propre cohésion », op. cit., p. 19. mité (Chamboredon et Lemaire, 1970) ${ }^{4}$ Bien qu'issues de la sociologie urbaine, ces analyses peuvent inspirer une lecture des reconfigurations sociales à l'œuvre lorsque les conflits d'usage autour de ressources territoriales rendent de plus en plus mouvante une action publique organisée autour de compromis instables entre groupes d'intérêts (Lascoumes, 1995). Elles permettent en outre, des rapprochements féconds avec les approches de la proximité développées en économie.

En effet, nous considérons également que les développements récents des analyses de la proximité en économie (Bellet et al., 1998 ; Gilly et Torre, 2000 ; Pecqueur et Zimmerman, 2004 ; Bouba-Olga et al., 2008), établis à partir du diptyque proximité d'essence spatiale (proximité géographique ou spatiale) / proximité d'essence non spatiale (proximité organisée ou socioéconomique), fournissent une base théorique féconde pour relire les phénomènes de conflictualité. Si l'on dresse un bilan des recherches proximistes réalisées sur les conflits d'usage et de voisinage, la majorité des travaux présente la proximité géographique comme un facteur polémogène et donc comme ayant un rôle dans l'apparition de ce type de conflit. À l'opposé, la fonction régulatrice de la proximité organisée est mise en avant, en insistant sur son rôle dans la résolution de la conflictualité (Torre et Caron, op. cit. ; Granjou et Garin, 2006).

L'objectif de notre article s'inscrit en complémentarité de ces travaux à travers l'appréhension de la nature ambivalente de la notion de proximité organisée. Nous proposons en effet d'analyser ses effets négatifs

4. «La diversification de sous-groupes nettement particularisés, l'éclatement des catégories de perception coutumières, la disparition de l'unité de voisinage comme instance qui rappelle et contrôle le respect des normes du groupe, la diversité conséquente des groupes de référence possibles [...] tout concourt à encourager, dans certaines catégories, [...] des aspirations à la mobilité [...] », op. cit., p. 33. 
possibles et plus particulièrement en quoi la résolution des conflits d'usage et de voisinage peut être conditionnée par le passage d'une proximité organisée polémogène à une proximité organisée régulatrice de conflits (Torre et Zuindeau, 2009). Nous proposons plus précisément de traiter cette question à partir de la grille proximiste, en intégrant une distinction au niveau de la proximité organisée, entre la logique intra-groupe et la logique inter-groupe. Nous montrerons alors qu'il existe une pluralité de configurations évolutives de la proximité organisée qui va avoir des effets, d'une part sur le niveau de la conflictualité, et d'autre part sur la résolution des problèmes d'externalités.

Dans une première partie, nous analysons la situation conflictuelle observée sur le littoral charentais, d'une part entre agriculteurs et conchyliculteurs, et d'autre part entre conchyliculteurs et plaisanciers. Notre terrain d'étude nous permet ainsi de mettre en évidence le pouvoir explicatif des notions proximistes à travers une analyse dynamique des relations entre acteurs. Nous nous centrons ensuite sur le rôle de la proximité organisée en ce qui concerne les possibilités de résolution des conflits d'usage et de voisinage observés.

\section{Analyse du conflit agriculteurs/conchyliculteurs}

La Région Poitou-Charentes, territoire rural mais attractif ${ }^{5}$, présente un fort contraste entre sa frange littorale, attractive et densément peuplée, et la zone intérieure, rurale et faiblement peuplée. Le littoral charentais s'étend sur $460 \mathrm{~km}$ de côtes et $150 \mathrm{~km}$ de littoral que contient la Charente-Maritime, seul département maritime de la région. $\mathrm{La}$ zone possède un climat agréable en raison d'un ensoleillement important et d'une faible pluviométrie.

5. C'est la 7e région la plus attractive de France, avec 12500 habitants supplémentaires entre 1999 et 2008, dont 11900 sont le fait du solde migratoire.
Trois activités économiques dominent : l'agriculture $^{6}$, le tourisme ${ }^{7}$ et la conchyliculture $^{8}$, toutes trois fortement dépendantes de la ressource en eau. L'agriculture exerce des pressions à la fois quantitatives (volumes prélevés pour les cultures irriguées) et qualitatives (pollutions liées aux produits phytosanitaires). La conchyliculture, activité non préleveuse, est cependant dépendante de l'état (qualitatif et quantitatif) de la ressource en eau (croissance des coquillages conditionnée par le degré de salinité de l'eau). L'eau est enfin un vecteur d'attractivité touristique. L'organisation territoriale du littoral charentais est marquée par des dysfonctionnements, en lien avec des phénomènes de concurrences et d'externalités négatives, qui ont conduit à l'émergence de conflits d'usage et de voisinage autour de la ressource en eau (Bouba-Olga et al., op. cit.). Nos investigations de terrain montrent qu' initialement le premier conflit concerne le collectif des agriculteurs et celui des conchyliculteurs autour de l'état de la ressource en eau en tant que bien linéaire (majoritairement matérialisé par le fleuve Charente). Pour comprendre comment le conflit est apparu et comment les acteurs se sont organisés pour défendre leurs intérêts, il est nécessaire de faire un rapide historique des deux activités sur le territoire étudié.

6. Chiffre d'affaires de 3 milliards d'euros, parmi les quatre premières régions productrices de céréales (Agreste, 2011).

7. Le Poitou-Charentes est la 7e région touristique, avec plus de 2,5 milliards d'euros de consommations touristiques (Mémento du Tourisme, 2010). La Charente-Maritime est le deuxième département au niveau de la fréquentation touristique.

8. Première région conchylicole française au niveau du tonnage, du nombre de sièges d'entreprises et du nombre d'emplois. Le bassin Marennes-Oléron est le plus important d'Europe. Deux activités présentes : ostréiculture et mytiliculture. Le chiffre d'affaires de l'ostréiculture, réalisé par $95 \%$ des entreprises du bassin, est de 250 millions d'euros en 2007. 


\section{1. Évolution des activités agricoles et conchylicoles}

À force d'une lutte incessante et séculaire contre les éléments terrestres et marins, les hommes sont, dans le passé, parvenus à instaurer une gestion complémentaire entre les eaux douces et salines leur profitant socio-économiquement. Cet équilibre provient du fait que la majorité des familles pratiquait à la fois l'agriculture et la conchyliculture sur le littoral charentais, les marais salés et l'estran ${ }^{9}$ étant consacrés à la conchyliculture et à la pêche littorale alors que les marais doux et les coteaux abritaient majoritairement la céréaliculture. Pour comprendre la situation actuelle sur ces territoires contigus et les conséquences qui en résultent, notamment au niveau de la nature des interactions et des relations entre les deux collectifs d'acteurs, il est nécessaire d'appréhender l'enchainement des évolutions de chacune des deux activités lors des cinquante dernières années.

Au cours des années 1970, l'ostréiculture connaît une importante épizootie qui anéantit la totalité du cheptel. Cette crise conduit à l'élimination des producteurs les plus fragiles économiquement et impose à ceux qui font le choix de poursuivre leur activité de se moderniser et par conséquent d'investir dans la mécanisation et dans un nouveau cheptel (huîtres d'origine japonaise). Pour réaliser cette mutation, les ostréiculteurs sont contraints de recourir tout à la fois à leur épargne, au Crédit Maritime et à la vente d'une partie de leurs biens fonciers (à des familles qui font le choix de se spécialiser sur l'agriculture). L'autre activité conchylicole, la mytiliculture, connaît une évolution assez similaire. La forte mortalité observée à la fin des années 1950, couplée à un allongement du temps de culture, obligent les professionnels à déplacer leur zone de production (d'où une hausse du temps de déplacement). Là

9. Zone littorale comprise entre le niveau haut et le niveau bas des marées. encore, ceux qui veulent continuer l'activité mytilicole sont obligés de se consacrer exclusivement à cette activité et de délaisser l'activité agricole. Se faisant, les conchyliculteurs se voient dépossédés des zones les plus en amont et donc de leur gestion.

Dès le début des Trente Glorieuses, l'agriculture vit de profondes mutations professionnelles, en lien avec une dynamique nationale (politique agricole) et des faits plus locaux. D'une part, les cessations d'activité des exploitants âgés, principalement ceux détenteurs de petites structures foncières exploitées de façon relativement extensive, profitent aux entrepreneurs agricoles. D'autre part, l'intensification agricole s'impose, à travers différentes phases concernant la maitrise de l'eau (en lien avec le développement de l'irrigation) et avec des conséquences importantes sur les marais salés et l'estran.

- Le premier événement concerne l'édification du barrage-écluse de Saint-Savinien en 1961 dans le but de stopper la salinité vers l'amont due aux marées et donc d'exploiter plus intensivement les terres de vallée. Le canal Charente-Seudre permet d'assurer l'alimentation des marais doux situés entre Tonnay-Charente et Marennes en période d'étiage.

- Le second événement consiste - lorsque cela est possible techniquement - à remplacer les anciennes portes de gestion des eaux de marais par des portes à crémaillères ou par des pompes à haut débit. La profession obtient par ce biais le contrôle de la gestion de l'eau des marais doux.

- Le troisième événement est la création du domaine expérimental de l'INRA au cœur du marais rochefortais à la fin des années 1970, dont l'objectif est de développer la mise en culture des marais grâce à de nouvelles techniques de drainage et de marnage des sols. Au total, le développement agricole se réalise sur la base d'un modèle économique n'intégrant pas les 
conséquences de ses externalités négatives et entrainant une gestion déséquilibrée de l'eau entre les acteurs du territoire.

\section{Conséquences au niveau de l'occupation de l'espace}

En conséquence des évolutions qui viennent d'être décrites, un découpage du territoire littoral s'opère au niveau de l'occupation des sols entre les agriculteurs et les conchyliculteurs. Pendant que les premiers cultivent les terres agricoles, les seconds exploitent les concessions qui leur ont été attribués sur l'estran charentais. Les deux collectifs sont donc proches au niveau géographique et reliés par la ressource en eau, matérialisée par le fleuve Charente et ses affluents. Ainsi, les acteurs en amont, les agriculteurs, impactent les acteurs en aval, les conchyliculteurs, à travers les pressions exercées sur la ressource en eau (modification des écoulements naturels et usage d'intrants chimiques). Le processus de production conchylicole nécessite en effet le mariage équilibré entre eaux douces et eaux salines à deux périodes de l'année, de juin à août au moment de la reproduction, et de novembre à janvier au moment de l'affinage. Or cet équilibre est directement lié à l'arrivée des eaux issues de l'amont. De leur côté, les céréaliers des coteaux, et encore davantage des marais doux littoraux, cherchent à rejeter les eaux pluviales hivernales et à conserver des volumes suffisants en période estivale afin d'alimenter leurs cultures. Les besoins des deux activités, agriculture et conchyliculture, vis-à-vis de la ressource en eau sont donc diamétralement inverses ${ }^{10}$.

Les intérêts divergents entre les agriculteurs et les conchyliculteurs conduisent à une situation conflictuelle, cas typique de conflits d'usage et de voisinage. Si la proximité géographique peut être qualifiée de forte, elle

10. S'ajoutent à cette opposition des besoins au niveau quantitatif, des problèmes au niveau qualitatif que nous ne développons pas ici. est subie par les acteurs en présence de par leur activité économique. Ils sont localisés dans des lieux spécifiques qui permettent l'exercice de leur activité (activité agricole fortement reliée à la terre et activité conchylicole fortement reliée à la mer) où ils ont réalisé des investissements spécifiques, qui sont par définition peu redéployables. De plus, les conchyliculteurs situés dans la baie de Marennes-Oléron bénéficient de l'indication géographique protégée (IGP) «Huîtres Marennes-Oléron ». De ce fait, les deux collectifs envisagent difficilement une modification de leur localisation en tant que solution effectivement réalisable pour ne plus avoir à subir le dysfonctionnement, étant donné le coût d'opportunité et les difficultés techniques qu'induirait une délocalisation de leur activité. Les agriculteurs et les conchyliculteurs sont donc pour la plupart en situation de lock-in (enfermement) spatial.

Enfin, les deux groupes d'acteurs se trouvent en situation d'inégalité face à l'espace. Les agriculteurs sont favorisés par leur situation géographique en amont, par rapport à la ressource qui fait conflit et par rapport aux conchyliculteurs. Cette asymétrie a des conséquences sur les relations entre les collectifs en présence. Les agriculteurs, de par leur position géographique avantageuse, n'ont en effet pas intérêt à entrer en conflit pour modifier l'ordre des choses, étant donné qu'ils n'ont pas à souffrir des pressions éventuelles exercées sur l'eau par les conchyliculteurs. À l'opposé, ces derniers, de par leur vulnérabilité liée à leur position géographique en aval, vont être incités à entrer en conflit pour défendre leurs intérêts, modifier la situation en place et ne plus voir leur activité économique subir les effets des pressions agricoles.

\section{Conséquences au niveau de la coordination entre acteurs}

À l'origine, il existait une forte proximité organisée entre agriculteurs et conchyliculteurs, liée au partage de valeurs communes, à leur pluriactivité et à leurs connaissances 
communes des marais doux et salés. En plus de l'organisation spatiale du territoire, la segmentation des acteurs en deux professions disjointes impacte les relations socioéconomiques sur ce même territoire. Mais, malgré les tensions et les conflits qui peuvent opposer les deux collectifs, le partage de ces valeurs communes et le sentiment d'appartenir à la même profession (paysans de la terre $v s$. paysans de la mer) a pour effet de ne jamais stopper la discussion.

La nature de la coordination entre les deux collectifs, agriculteurs et conchyliculteurs, est également marquée par une asymétrie institutionnelle. Il existe en effet une relation privilégiée entre la profession agricole et l'Administration, notamment à travers la politique d'intensification agricole développée au lendemain de la Seconde Guerre mondiale. Dans cette perspective, à titre d'exemple, le Conseil Général participe financièrement aux aménagements hydrauliques ainsi qu'à la création de l'Union des marais de la Charente-Maritime (UNIMA) et aide financièrement à partir des années 1980 au développement de l'irrigation des terres hautes qui entourent les marais doux et bordent les fleuves. À l'opposé, la conchyliculture est à l'époque considérée comme une activité marginale en raison de son passé et de la rudesse de ses conditions de production. Mais les dernières décennies ont vu cette activité se moderniser pour devenir un secteur productif de renom national et international, tout en gardant son caractère individualiste. Les deux activités sont régies par le même Ministère, mais on observe des disparités entre les deux collectifs au niveau de la représentation institutionnelle. Les conchyliculteurs n'ont pas de chambre dédiée à leur activité et cotisent, tout comme les agriculteurs, à la chambre d'Agriculture de leur département. Mais, à l'opposé des agriculteurs qui peuvent s'appuyer sur des représentants, les conchyliculteurs n'ont pas de service spécifiquement dédié à leur activité pour les représenter. Ainsi, même s'ils possè- dent des organisations professionnelles du type syndicats des bans ${ }^{11}$ et Section régionale conchylicole (SRC), leur représentativité départementale ${ }^{12}$, régionale et nationale est faible au regard du syndicat majoritaire - la Fédération nationale des syndicats d'exploitants agricoles (FNSEA) - aux fortes implications départementales.

Le déséquilibre entre les deux collectifs commence à s'atténuer depuis 2004, les deux professions étant mieux représentées au sein des institutions. À cette date, le président de la SRC est en effet élu vice-président de la Région Poitou-Charentes et chargé des problématiques « Agriculture, Cultures marines, Pêche et Littoral ». Cette élection se déroule, d'une part, dans un contexte marqué par la montée en puissance des politiques environnementales, notamment en ce qui concerne la gestion de l'eau, et d'autre part, au moment précis où les critiques sociales à l'encontre des pratiques agricoles se multiplient. Elle a également lieu au lendemain d'une année de canicule pendant laquelle la conflictualité entre les deux professions s'est aggravée et où d'importantes manifestations conchylicoles estivales ont perturbé la saison touristique. Enfin, elle offre au monde conchylicole une reconnaissance institutionnelle au sein de la sphère politique et productive départementale, de sorte qu'il devient un acteur inévitable dans toutes décisions concernant la gestion de l'eau et notamment lors de l'élaboration des SAGE (Schéma d'aménagement et de gestion des eaux) des fleuves Charente et Seudre. Les étés très secs observés de 2005 à 2009, et leurs consé-

11. Espaces sur l'estran agréés pour la production d'huîtres et de moules.

12. Le Conseil général de la Charente-Maritime a eu et continue d'avoir comme président et comme conseillers des représentants du monde agricole (tels que A. Dulin, F. Blaizot ou encore D. Bussereau.). À l'opposé, aucun élu n'avait pour profession celle de conchyliculteur ce qui participe à la faible audience de la profession au sein des instances décisionnelles départementales. 
quences sur la production conchylicole, crédibilisent également le discours de la SRC. Les professionnels conchylicoles réalisent des actions d'envergure (exemples des manifestations escargot sur des axes très fréquentés tel que le pont de l'île d'Oléron), en utilisant les médias et les inquiétudes de l'opinion publique concernant l'environnement, pour sensibiliser les populations et les gagner à leur cause. Les conchyliculteurs ont d'ailleurs réussi par leurs actions, à travers une décision du préfet, à faire procéder à des lâchers d'eau douce et à la mise en place de sondes pour connaître la salinité de l'eau ${ }^{13}$. Enfin, la SRC met en avant la nécessité de défendre une activité historiquement localisée dans la région et labellisée mais aussi son poids sur les marchés nationaux et internationaux.

Au total, en l'espace de quelques années s'opère un rééquilibrage des rapports de force institutionnels entre les mondes agricole et conchylicole. Face à cette réalité et aux critiques sociales, l'agriculture se retrouve en position d'accusée et dans l'obligation de faire évoluer son discours et ses pratiques.

\section{Analyse du conflit conchyliculteurs/plaisanciers}

Plus récemment, un autre collectif est apparu dans ce système conflictuel. Les plaisanciers s'opposent eux aussi aux conchyliculteurs, mais cette fois au niveau de l'usage de l'espace de navigation charentais.

\section{1. Évolution des activités conchylicoles et de nautisme/plaisance}

Pour appréhender l'évolution du système d'interdépendance propre au littoral charentais et celle de la situation conflictuelle qui en découle, il est nécessaire d'intégrer les

13. Le Centre régional de recherche et d'application aquacole (CREAA) démontre, à l'aide des suivis hydrologiques effectués, l'absence d'arrivée d'eau douce à l'embouchure de la Charente ; réalité jusqu'alors niée par le monde agricole. problématiques productives conchylicoles plus récentes. Depuis une dizaine d'années, les conchyliculteurs sont en effet confrontés à un problème de productivité, lié à la surcharge de l'estran (densité de coquillages trop forte sur l'estran qui a pour conséquence une diminution des potentialités de production et un allongement de la durée du cycle de production) et aux problèmes autour de la ressource en eau. Pour tenter de répondre à ces difficultés, de nombreuses innovations productives apparaissent : naissain d'écloserie, huître triploïde, production sur filières. Ces innovations permettent, surtout si elles sont combinées, d'assurer la quantité et la taille des huîtres produites tout en réduisant la durée du cycle de production et les contraintes liées au calendrier de production.

La production sur filières ${ }^{14}$ se développe sur le littoral charentais, impulsée au départ par un ostréiculteur de la région, M. Marissal, qui a développé cette nouvelle technique de production aux débuts des années 1990. Le préfet autorise l'installation de filières ostréicoles expérimentales en 1992 au nord de l'Île de Ré, en même temps qu'un plan de filières mytilicoles. Ce nouveau procédé permet d'une part d'augmenter la productivité conchylicole, et d'autre part de relâcher la contrainte eau de l'activité (production plus éloignée de l'estran). Les filières permettent de soulager quelque peu la conflictualité entre les deux collectifs mais ne constituent qu'une réponse incomplète au problème de partage de la ressource sur le territoire concerné. De plus, leur développement demande une évolution importante aux niveaux des compétences à mobiliser et reste circonscrit aux entreprises les plus importantes sur le plan économique du fait des investissements nécessaires. En 2000, les mytiliculteurs

14. Filins tendus en mer avec des lignes suspendues où sont fixés les mollusques. Technique qui impose la création de champs d'élevage et la définition d'aires maritimes réservées. 
souhaitent développer des filières dans la baie d'Yves. Leur installation débute fin 2006, alors que la baie de la Malconche (entre l'Île d'Oléron et l'Île d'Aix) est pressentie pour l'installation d'un autre projet de filières. En 2007, le plan de filières au nord de l'île de Ré voit sa surface doubler, atteignant les $12 \mathrm{~km}^{2}$.

Dans le même temps, l'activité de plaisance se développe fortement en France, et plus particulièrement en Charente-Maritime. La filière nautique est une activité phare de la Région Poitou-Charentes ${ }^{15}$. Le premier port de plaisance du littoral atlantique (port des Minimes de La Rochelle), ajouté au plan d'eau protégé des Pertuis ${ }^{16}$, donne un vrai avantage à la région au niveau de l'attractivité des plaisanciers. Dans le passé, l'activité de plaisance était assez individualiste et il n'existait pas de « collectif » plaisancier à l'échelle départementale attaché à défendre ses intérêts. Mais, face à la multiplication des projets conchylicoles, le besoin de se défendre collectivement et de manière structurée augmente. C'est ainsi qu'en 2001 une union départementale des plaisanciers est créée en Charente-Maritime, à l'origine de I'UNAN (Union nationale des associations de navigateurs). Dès 2003, l'UNAN adresse des courriers au préfet pour alerter sur le risque de mitage des pertuis charentais par les filières conchylicoles.

\section{Conséquences au niveau de l'occupation de l'espace}

Les évolutions propres à chacun des deux collectifs, conchyliculteurs et plaisanciers, vont influer sur les relations entre les acteurs

15. Ce secteur industriel regroupe 430 entreprises en Poitou-Charentes pour un chiffre d'affaires de l'ordre de 400 millions d'euros en 2007 et emploie 4000 salariés (chiffres clés du nautisme 20062007, Fédération des industries nautiques).

16. Les Pertuis permettent des destinations de courtes durées (croisières courtes) qui accordent une sortie au week-end. Sur toute la façade atlantique, il n'y a que deux lieux qui autorisent cela : les Pertuis charentais et la baie de Quiberon. du territoire. En effet, les conchyliculteurs modifient la localisation géographique de leur zone de production puisque ceux qui utilisent les filières produisent désormais plus loin en mer (par rapport à la localisation traditionnelle sur l'estran) et se trouvent sur l'espace de navigation charentais emprunté par les plaisanciers lors de la pratique de leur activité de loisir. On observe ainsi une modification de l'occupation de l'espace maritime, à l'origine d'un nouveau conflit d'usage, toujours autour de la ressource en eau, mais cette fois en tant que bien surfacique (l'eau est appréhendée en tant qu'espace), entre les conchyliculteurs et les plaisanciers. Les deux collectifs s'opposent autour de l'usage de l'espace maritime, les plaisanciers estimant que les filières représentent une appropriation privée d'un espace par définition public. Ces derniers mettent également en avant le fait que les filières représentent un danger pour la navigation en mer, un risque environnemental (exemple de l'envasement de la plage de l'île de Noirmoutier depuis l'installation de filières), et l'absence d'étude mesurant leur impact sur le plan économique.

Cette opposition matérialise un conflit de superposition, étant donné que les deux groupes d'acteurs localisés sur le même territoire s'opposent quant à l'usage à développer sur le territoire en question. L'eau, en l'occurrence la mer, est ainsi à la fois un support favorable à une diminution de la vulnérabilité de la conchyliculture au regard des pressions exercées par les activités terrestres mais constitue également un enjeu d'occupation de l'espace (Bouba-Olga et al., op. cit.). Ce « nouveau » conflit d'usage est donc en fait une extension sociale et spatiale du conflit plus global autour de la ressource en eau.

Les deux collectifs sont donc là encore caractérisés par une proximité géographique forte et subie, c'est d'ailleurs ce qui forme l'origine de la situation conflictuelle. Nous avons déjà précisé l'importance de la ressource en eau pour les conchyliculteurs et 
en quoi elle contraignait fortement la localisation des professionnels. Des conchyliculteurs ont tout de même réussi, au prix d'innovations coûteuses en termes d'investissements, à s'éloigner quelque peu de l'estran. Les plaisanciers suggèrent ainsi que la solution au conflit qui les oppose aux conchyliculteurs serait un éloignement plus important de l'estran par la mise en place de filières offshore (en pleine mer), au large des pertuis, là où elles gêneraient moins la navigation. Ceci semble cependant difficilement envisageable, étant donné les coûts à engager (investissements importants, augmentation du risque lié aux tempêtes, coût du déplacement) et les compétences à mobiliser (développement de connaissances de navigation maritime). Si on assimile la plaisance à une activité de tourisme, nous sommes tentés de considérer que la proximité géographique est alors recherchée et qu'un déplacement de l'activité est facilement envisageable. Mais en intégrant les problématiques internes à l'activité de nautisme-plaisance, nous développons une analyse plus rigoureuse et plus réaliste de la situation d'interdépendances. Du fait de la spécificité du bassin de navigation charentais, les plaisanciers sont en effet peu incités à envisager une délocalisation de leur activité de loisir. À ceci s'ajoute un phénomène de saturation des ports aux niveaux national et européen. Ainsi, les plaisanciers se trouveraient plutôt dans une situation de proximité géographique subie, étant donné leur incapacité à se localiser dans un autre port.

\section{Conséquences au niveau de la coordination entre acteurs}

Les premiers plans de filières conchylicoles ne font pas l'objet d'opposition franche des plaisanciers étant donné leur absence de cohésion et d'organisation à cette époque. Face à l'initiative conchylicole, ils n'offrent pas au départ de front uni capable d'engager une démarche collective pour la défense de leurs intérêts. Mais avec la multiplication des projets, leur crainte de voir leur espace de navigation envahi par les filières augmente, d'où la nécessité grandissante pour eux de s'organiser en collectif.

Cette opposition au niveau de l'usage des pertuis charentais s'ajoute à une proximité organisée relativement faible entre les deux collectifs, couplée à une asymétrie institutionnelle. Les plaisanciers forment un collectif plus volatile (origines diverses, pas obligatoirement issus de Charente-Maritime ou de la région) et moins mobilisateur. Ils sont ainsi perçus par l'Administration et les conchyliculteurs comme des nantis pratiquant une activité de loisir ${ }^{17}$ et les professionnels considèrent de ce fait qu'ils sont prioritaires sur eux. À cela s'ajoute le soutien de l'Administration accordé à la profession conchylicole, à travers les Affaires Maritimes et le Ministère chargé de l'agriculture et de la pêche (création d'une mission interministérielle pour la conchyliculture qui a abouti à la mise en place du Comité de coordination des politiques littorales). En raison des enjeux économiques de ce secteur d'activité, la conchyliculture reçoit le soutien de son Administration au détriment de l'activité de plaisance. Les plans de filières sont dans ce cadre perçus comme des conditions favorables au maintien de l'activité sur le territoire. La reconnaissance institutionnelle grandissante de la SRC et l'influence de son président jouent également de manière certaine lors des négociations avec l'Administration. De plus, les conchyliculteurs font preuve d'une facilité à mobiliser et à manifester pour défendre leurs intérêts, alors que les plaisanciers ont une capacité de nuisances plus faible. On observe ainsi entre les deux collectifs une situation inverse à celle présentée entre agriculteurs et conchyliculteurs : ce sont cette fois les conchyliculteurs qui bénéficient d'un pouvoir d'antériorité quant à leur représentation et à la défense de leurs intérêts puisqu'ils sont structurés depuis plus longtemps que les plaisanciers et qu'ils

17. Source : entretien avec un représentant de l'activité de plaisance. 
ont un pouvoir de lobbying plus fort. Les plaisanciers tentent d'augmenter leur pouvoir de négociation auprès de l'Administration en s'alliant avec d'autres acteurs, d'une part avec les acteurs de la filière nautique, représentée par la Fédération des industries nautiques (FIN) et largement présente autour de La Rochelle, et d'autre part avec les marins pêcheurs, eux aussi directement concernés par l'extension des filières dans les pertuis.

La position institutionnelle dominante des conchyliculteurs vis-à-vis des plaisanciers et la faiblesse de la proximité organisée sont à l'origine du contentieux juridique concernant les filières de la baie d'Yves ${ }^{18}$. Ce contentieux illustre bien l'inégalité institutionnelle entre les deux collectifs, puisque les Affaires Maritimes et le Ministère défendent ce projet aux côtés des conchyliculteurs et face aux plaisanciers.

\section{Les différentes configurations de proximité organisée}

L'observation des conflits autour de la ressource en eau sur le littoral charentais à travers le prisme proximiste nous a permis de rendre compte des logiques internes à chaque collectif impliqué (agriculteurs, conchyliculteurs et plaisanciers) et d'élaborer une analyse dynamique du système d'interdépendances en place sur le territoire. Ces éléments nous conduisent également à mettre en avant la complexité du rôle de la proximité organisée, à travers une prise en compte de sa possible dimension négative.

\section{L'ambivalence de la proximité organisée}

On considère traditionnellement que la proximité géographique possède un caractère polémogène et que la proximité organisée possède

18. Les plaisanciers estiment, malgré un premier déplacement obtenu après concertation avec les conchyliculteurs, que les filières installées dans la baie d'Yves sont mal placées, car situées sur la route de navigation très fréquentée reliant $\mathrm{La}$ Rochelle à l'île d'Aix. Cette opposition entre plaisanciers et conchyliculteurs a mené à un contentieux juridique (Boutry O., 2010). davantage un rôle de facilitateur de relations entre les acteurs et donc un rôle davantage d'apaisement des conflits. Cette dernière participerait à la recherche de compromis à travers son rôle dans la production et/ou l'acceptation de règles de gestion. Il est ainsi supposé que le développement de la proximité organisée participe à la prévention ou à la gestion des conflits étant donné qu'elle favorise la coordination entre les acteurs vers la recherche d'une solution négociée. Cette idée nous semble hâtive et restrictive, le rôle de la proximité organisée étant selon nous plus complexe. En effet, cette dernière peut également participer à l'intensification du conflit et bloquer les tentatives de recherche de solutions négociées. Ainsi, nous cherchons à appréhender son rôle ambivalent pour comprendre les possibles situations de blocages en ce qui concerne la recherche de solutions à des problèmes d'externalités à l'origine de situations conflictuelles.

La résolution des conflits d'usage et de voisinage pose la question de l'action collective (Ostrom, 1992, 1998), elle-même liée aux formes de coordination entre acteurs. À cet égard, la sociologie de l'action organisée nous rappelle que l'intégration d'individus dans un collectif se traduit souvent par des compromis instables qui n'excluent pas la coexistence d'intérêts contradictoires et qui menacent constamment la cohésion interne du groupe soumis à la perspective centrifuge des reconfigurations d'alliances (Crozier et Friedberg, 1977) ${ }^{19}$. Dans ce cadre, la situation des acteurs dans l'espace physique et dans l'espace socio-économique impacte leurs processus de décisions. C'est pourquoi nous considérons qu'il est pertinent d'analyser les liens entre les individus en distinguant le niveau individuel et le niveau collectif. La proximité organisée peut ainsi être observée

19. "Les construits d'action collective [...] organisent des modes d'intégration qui assurent la nécessaire coopération entre acteurs sans supprimer leurs libertés, c'est-à-dire de poursuivre des objectifs contradictoires », op. cit., p. 22. 
à deux niveaux, l'intra-groupe et l'intergroupe. Cette distinction est primordiale pour comprendre le rôle ambivalent de la proximité organisée. Dans le premier cas, la proximité renvoie à l'appartenance ou non à un même collectif et aux valeurs partagées en son sein. La seconde logique, la logique inter-groupe, fait, quant à elle, référence aux liens entre les collectifs formés. On observe dans de nombreuses situations conflictuelles, une segmentation des acteurs concernés en différents collectifs dans le but d'avoir davantage de poids dans le conflit et de défendre leurs intérêts, mais cette organisation en collectif ne garantit en rien la résolution d'une situation conflictuelle. En effet, à travers l'existence d'une forte proximité organisée intra-groupe, la construction de collectif peut conduire à une forme de radicalité et bloquer toute tentative de relations entre les collectifs. Au niveau individuel, la proximité organisée permet donc de faciliter la coordination avec d'autres acteurs, à travers des dispositifs de mise en relation des acteurs. Mais ces dispositifs vont également impacter les relations au niveau supérieur, c'est-à-dire entre les collectifs d'acteurs. Ainsi, la compréhension des relations entre les collectifs d'acteurs formés dans le cas des conflits d'usage et de voisinage nécessite d'analyser les dispositifs de mise en relation internes aux collectifs. On peut observer plusieurs configurations évolutives de la proximité organisée sur un territoire, qui renvoient à la manière dont les individus, et les collectifs, se coordonnent. Ces différentes configurations, liées aux formes de proximité organisée intra-groupe et inter-groupe rencontrées, vont conditionner le niveau de la conflictualité et les possibilités de résolution des conflits d'usage et de voisinage sur les territoires.

\section{La construction d'une proximité organisée intra-groupe}

Sur le littoral picto-charentais, on a pu observer, au niveau de chaque collectif, l'émergence d'une proximité organisée à travers le regroupement des acteurs dans le but de défendre leurs intérêts communs.
Chaque collectif s'est, à des périodes différentes, recentré sur ses valeurs, ses objectifs et sa perception de la situation. On observe ainsi, sur le littoral charentais, trois collectifs. Le premier, le plus ancien correspond au monde agricole. Le second renvoie au monde conchylicole. Enfin, le troisième, le plus récent et le moins structuré, est composé de la plaisance et de la filière nautique.

Dans les années 1970, le collectif agricole est très puissant. Ses membres bénéficient d'un pouvoir de mobilisation, d'un avantage certain au niveau de leur représentativité et du soutien de l'Administration. Ils jouissent d'une proximité organisée forte. Cette configuration, caractérisée par une asymétrie entre agriculteurs et conchyliculteurs au niveau de la proximité intra-groupe et par une faible proximité inter-groupe, conduit à un niveau de conflictualité faible et à la non résolution du problème d'externalité autour de la ressource en eau.

On observe ensuite, au début des années 2000, la structuration des conchyliculteurs en collectif. Ceux-ci gagnent ainsi en pouvoir et en crédibilité et tentent de défendre plus efficacement leurs intérêts et d'avoir davantage de poids sur le territoire. Ainsi, si la proximité organisée semble toujours relativement faible entre les collectifs, elle apparaît à l'opposé comme d'autant plus forte au sein de chacun de ces deux collectifs. Cette configuration de proximité organisée ne permet pas d'apaiser les relations entre acteurs et d'aider à la mise en place de règles communes acceptées par tous. En même temps que les rapports de force s'équilibrent progressivement, c'est « une course à l'armement » qui s'engage, à travers la recherche d'une part, de soutiens parmi l'administration, les collectivités publiques et le monde politique, et d'autre part, de preuves scientifiques de l'existence des externalités. On a ainsi pu observer par le passé une multiplication d'études scientifiques - le plus souvent concernant la détermination des impacts des pressions 
exercées sur la ressource en eau, et notamment les pressions agricoles, sur la productivité conchylicole, - et la confrontation de ces résultats, souvent différents, lors des tentatives de négociations ${ }^{20}$. Chaque collectif finance donc des études et reste campé sur ses positions. La conjonction d'une proximité organisée forte au sein de chaque collectif (agricole et conchylicole), couplée à une proximité faible entre ces mêmes collectifs, provoque une montée du niveau de la conflictualité et un blocage au niveau de la recherche d'une solution négociée. Chacun se recentre sur son collectif et cela conduit à une montée du degré de conflictualité sur le littoral charentais, et à un blocage en ce qui concerne la résolution du problème d'externalités au niveau de la ressource en eau.

\section{Vers une proximité organisée inter-groupe ?}

Des évolutions plus récentes se manifestent, liées à des pressions politiques, sociales (revendications sociales et rôle de l'opinion publique notamment) et économiques, et celles-ci participent à une montée de l'hétérogénéité interne aux collectifs. Cette hétérogénéité croissante, observée depuis ces dernières années, a pour effet de diminuer leur pouvoir de représentation et complique la conduite des négociations sur le littoral picto-charentais.

Le monde agricole évolue sous l'effet d'une prise de conscience plus ou moins collective à deux niveaux. Le premier renvoie à l'image à donner aux autres acteurs et plus globalement à la société. Les instances dirigeantes départementales agricoles ont en effet compris la nécessité de montrer à la société, et prioritairement aux autres acteurs institutionnels départementaux et régionaux, que la profession est, elle aussi, respectueuse de l'environnement et qu'elle modifie à cette

20. La profession agricole demandait aux conchyliculteurs des études scientifiques capables de prouver le lien existant entre l'état de la ressource en eau douce et les problèmes de productivité conchylicole. fin ses pratiques. Si la réalité n'est pas homogène, le discours est bien là, tant vis-à-vis de l'extérieur que dans les messages internes. Le second niveau, interne, est moins manifeste en raison des rapports de pouvoir existants au sein de la sphère agricole. On observe une segmentation au sein de la profession entre d'une part, l'agriculture « traditionnelle » et d'autre part, les éleveurs et les syndicats minoritaires opposés à l'irrigation des terres hautes et au drainage des marais doux. Les uns et les autres défendent la nécessité d'une autre gestion des ressources naturelles et de l'eau en particulier. Le collectif agricole apparait ainsi beaucoup moins homogène qu'il y a trente ans. De par leurs revendications, les éleveurs et les syndicats non irrigants rejoignent les attentes des conchyliculteurs et imposent à la majorité agricole de se repositionner.

Des rivalités internes à la conchyliculture existent également, liées à des perceptions différentes en ce qui concerne l'évolution de la profession, aussi bien au niveau de l'acte de production que de son ancrage territorial. Certains conchyliculteurs tentent de défendre les conditions habituelles de production sur l'estran et l'image d'un produit traditionnel et naturel. Ils sont ainsi très vulnérables vis-à-vis des pressions exercées sur la ressource en eau et se trouvent directement en conflit avec les agriculteurs irrigants. D'autres conchyliculteurs, plus jeunes et innovants, cherchent à échapper aux contraintes qui pèsent sur leur activité, en intensifiant les moyens de production afin d'augmenter la productivité. Ils recourent aux dernières innovations conchylicoles (notamment les filières conchylicoles et les huîtres triploïdes), et comptent d'importants entrepreneurs (du fait du niveau élevé d'investissements à engager). Ces innovations permettent de relâcher quelque peu la contrainte « eau » de l'activité conchylicole et de diminuer dans le même temps la conflictualité avec les agriculteurs. Ce faisant, « cette » conchyliculture suit la même logique de développement que la 
céréaliculture et entre en conflit avec les plaisanciers. On observe la constitution d'un clivage interne à la profession avec d'un côté, les petites structures qui produisent de manière traditionnelle, et de l'autre, les grosses structures qui recourent aux innovations conchylicoles pour produire de manière intensive. Cette segmentation des professionnels au sein de la profession conchylicole pourrait impacter les décisions concernant des futurs projets de filières.

Ces évolutions renvoient ainsi à une plus faible homogénéité des représentations et des comportements au sein des collectifs agricoles et conchylicoles (affaiblissement de la proximité organisée intra-groupe), et celle-ci semble participer à l'amélioration de la proximité organisée mais cette fois entre les collectifs impliqués. En effet, devant le poids grandissant des conchyliculteurs, la montée en puissance des revendications environnementales et les oppositions internes concernant le modèle de production agricole, les agriculteurs peuvent difficilement rester sur leurs positions et contester leurs responsabilités quant à l'état de la ressource en eau, aussi bien aux niveaux quantitatif que qualitatif. Ils font preuve d'une attitude plus conciliatrice et favorisent la recherche d'une solution négociée au conflit. Les signes apparents d'une diminution de l'homogénéité au sein du collectif agricole semblent ainsi contribuer à la résolution du problème d'externalité autour de la ressource en eau. Au total, cette nouvelle configuration de la proximité organisée, marquée par une proximité organisée intra-groupe forte, mais quelque peu déclinante, et une hausse de la proximité organisée inter-groupe, a donc pour conséquence de maintenir un certain niveau de conflictualité et d'engager les acteurs vers une résolution du problème d'externalités autour de la ressource en eau.

De manière analogue, on peut s'interroger sur les impacts des évolutions observées au sein du collectif conchylicole (clivages internes concernant le mode de production et ses conséquences sur la localisation spatiale de l'activité) sur l'issue du conflit avec les plai- sanciers. Ce conflit étant plus récent, il est plus difficile de se prononcer sur les évènements qu'on observe actuellement. La remise en cause, par certains professionnels, du mode de production sur filières (et des huîtres triploïdes) renvoie, comme nous venons de le décrire, à une diminution de la proximité organisée au sein du collectif conchylicole. Cette évolution, couplée à la construction du collectif plaisancier (hausse de la proximité organisée en son sein), aura peut-être des impacts sur le degré de conflictualité et la recherche d'une solution au problème de concurrence sur le domaine navigable charentais.

$$
\begin{gathered}
* * \\
*
\end{gathered}
$$

L'application de la grille proximiste au littoral charentais nous a permis de montrer les différentes configurations évolutives de la proximité organisée, liées à la manière dont les individus, et les collectifs, se coordonnent. Une analyse fine des positionnements, des contraintes et des stratégies des activités au cœur du conflit qui marque le littoral charentais nous amène en effet à prendre en considération un degré de complexité plus poussé de la dynamique conflictuelle du territoire. Nous avons également mis en évidence l'impact de ces configurations sur le niveau de la conflictualité et sur les possibilités de résolution des problèmes d'externalités sur le littoral observé.

Nous sommes ainsi en mesure d'appréhender d'une part, les conditions qui ont fait évoluer dans le temps les relations entre acteurs et qui ont participé à l'émergence des situations conflictuelles, et de mettre en avant d'autre part, les enjeux existants autour de la proximité organisée en ce qui concerne les possibilités de résolution. L'enjeu pour l'organisation territoriale du littoral charentais semble ainsi consister en une meilleure identification des évolutions actuelles du degré de proximité de coordination et des situations de blocage afin de faciliter l'émergence d'une logique de concertation et d'assurer une meilleure gestion de la ressource en eau sur le territoire. 


\section{RÉFÉRENCES BIBLIOGRAPHIQUES}

Bellet M., Kirat T., Largeron C. (1998). Approches multiformes de la proximité. Paris, Hermès.

Bossuet L., Torre A. (2009). Le devenir des ruralités, entre conflits et nouvelles alliances autour des patrimoines locaux. Economie rurale, $\mathrm{n}^{\circ} 313-314$, sept.-nov., p. 147-162.

Bouba-Olga O., Boutry O., Rivaud A. (2009). Un approfondissement du modèle exitvoice par l'économie de la proximité. Nature, Science et Société, vol. 17, $\mathrm{n}^{\circ} 4$, p. 381-390.

Bouba-Olga O., Carrincazeaux C., Coris M. (2008). La proximité 15 ans déjà ! Revue d'Économie Régionale et Urbaine, $\mathrm{n}^{\circ} 3$.

Boutry O. (2011). Agriculture et Environnement : une analyse néo-institutionnelle de l'évolution des pratiques agricoles - Le cas de la gestion quantitative de la ressource en eau en Charente-Maritime. Poitiers, Thèse pour le doctorat en Sciences Économiques,

Boutry O. (2010). D'un conflit à l'autre : les conchyliculteurs contre les plaisanciers. L'actualité Poitou-Charentes, $\mathrm{n}^{\circ} 89$, Numéro Spécial.

Chamboredon J.-C., Lemaire M. (1970). Proximité spatiale et distance sociale. Les grands ensembles et leur peuplement. Revue française de sociologie, vol. 11, $\mathrm{n}^{\circ}$ 1, p. 3-33.

Crozier M., Friedberg E. (1977). L'acteur et le système : les contraintes de l'action collective, Paris, Seuil, 500 p.

Elias N., Scotson J.-L. (1994 [1965]). The Established and the Outsiders. A Sociological Enquiry into Community Problems. London, Sage Publications.

Gilly J.-P., Torre A. (2000). Dynamiques de proximité. Paris, L'Harmattan.

Granjou C., Garin P. (2006). Organiser la proximité entre usagers de l'eau : le cas de la gestion volumétrique dans le Bassin de la Charente. Développement Durable et Territoires, dossier 7.

Lascoumes P. (1995). Les arbitrages publics des intérêts légitimes en matière d'environnement. L'exemple des lois Montagne et Littoral. Revue française de science politique, vol. 45, n 5, p. 396-419.

Nabli M. K., Nugent J. B. (1989). Collective Action, Institutions and Development. In Nabli M. K., Nugent J.-B., «The New Institutional Economics and Development. Theory and Application to Tunisia, Amsterdam, North-Holland », p. 80-137.

Ostrom E. (1992). Crafting Institutions for self-governing irrigation systems. San Francisco, ICS Pres, Institute for Contemporary Studies, $111 \mathrm{p}$.

Ostrom E. (1998). A behavioral approach to the rational-choice theory of collective action. American Political Science Review, vol. $92, \mathrm{n}^{\circ} 1$, p. 1-22.

Pecqueur B., Zimmermann J.-B. (2004). Économie de proximités. Paris, Hermès, $264 \mathrm{p}$.

Simmel G. (1981 [1908]). Sociologie et épistémologie. Paris, PUF.

Torre A., Aznar O., Bonin M., Caron A., Chia E., Galman M., Guérin M., Jeanneaux Ph., Kirat T., Lefranc C., Melot R., Paoli J.-C., Salazar M.I., Thinon P. (2006). Conflits et tensions autour des usages de l'espace dans les territoires ruraux et périurbain, le cas de six zones géographiques françaises. Revue d'Économie Régionale et Urbaine, $\mathrm{n}^{\circ} 3$, p. 415-453.

Torre A., Caron A. (2005). Réflexions sur les dimensions négatives de la proximité : le cas des conflits d'usage et de voisinage. Économie et Institutions, $\mathrm{n}^{\circ} 6 \& 7$, p. 183-220.

Torre A., Zuindeau B. (2009). Les apports de l'Économie de la proximité aux approches environnementales : inventaire et perspectives. Natures Sciences et Sociétés, vol. 17, p. 349-360. 